\title{
Facteurs et expression du polycyclisme juvénile chez le chêne rouge d'Amérique (Quercus rubra L) planté en conditions naturelles
}

\author{
A Cabanettes *, F Courdier, C Meredieu, P Trichet
}

INRA, station de recherches forestières, centre de Bordeaux, BP 45, 33611 Cestas-Gazinet cedex, France

(Reçu le 10 octobre 1994 ; accepté le 26 janvier 1995)

\begin{abstract}
Résumé - ll est montré, dans un essai installé en conditions naturelles sur un ancien sol agricole du sud-ouest de la France, que le chêne rouge d'Amérique (Quercus rubra $L$ ) est capable d'exprimer au stade juvénile plusieurs cycles de croissance (polycyclisme) dès la première année de plantation. $\mathrm{Ce}$ phénomène est apparu en 1992 uniquement si les plants étaient désherbés et n'a concerné qu'environ 1 tiers des plants désherbés. La fertilisation (NPK) ne semble pas avoir d'influence sur le polycyclisme. En revanche, ce sont les plants de plus petit diamètre initial et bénéficiant d'une alimentation en eau sans excès qui ont la plus grande probabilité de développer plus d'un cycle de croissance annuel. Le polycyclisme a eu pour conséquences une augmentation significative de l'accroissement en diamètre $(+130 \%$ ), de la ramification (qui passe de $24 \%$ en volume ligneux à $64 \%$ ), et de la surface foliaire $(+120 \%)$, et constitue de ce fait un avantage considérable pour la réussite de ce type de plantation. Son expression s'effectuant en partie sous forme de pousses-relais, il ne contribue pas à augmenter significativement la hauteur totale des plants la première année de leur installation.
\end{abstract}

\section{croissance / polycyclisme / ramification / surface foliaire / chêne rouge}

Summary - Factors and consequences of rhythmic growth on young American red oak in field conditions. In field conditions (ex-agricultural soils of south-west France), red oak is able to develop several growth flushes (polycyclism) in the 1st growing period of plantation. Polycyclism occurred only in the weeded treatment, and concerned one-third of weeded plants. NPK fertilization showed no influence on polycyclism. On the other hand, a significantly higher probability of polycyclism arose on small diameter plants or on plants without soil water excess. The consequences of polycyclism were an increase of diameter $(+130 \%)$, leaf area $(+120 \%$ ) and ramification rate (from 24 to $64 \%$ ). These advantages are very important for the success of this type of plantation. Polycyclic shoots were frequently basal shoots, and did not increase significantly the total tree height.

\section{growth / lamma shoots / leaf area / branching / American red oak}

* Adresse actuelle : INRA, unité d'agroforesterie, centre de Toulouse, BP 27, 31326 CastanetTolosan cedex, France 


\section{INTRODUCTION}

La croissance rythmique est une particularité de la plupart des arbres, que certains auteurs interprètent comme le résultat de l'association de méristèmes à des âges physiologiques différents sur le même individu (White, 1979). Elle se manifeste par l'alternance de phases d'allongement et de phases de repos ou de fort ralentissement selon une périodicité assez régulière caractéristique de l'espèce (Crabbé, 1987). Ce dernier point dénote un déterminisme endogène à base génétique. Toutefois, chez les espèces des régions à climat contrasté, on s'aperçoit que les caractéristiques de ce rythme sont en fait modulables par le milieu (variations selon l'année ou la méthode de culture). Ce fait est confirmé par les expérimentations en conditions contrôlées, qui montrent que les variations de l'éclairement, de la température ou de l'alimentation hydrique peuvent modifier l'amplitude et/ou la fréquence ou la durée du rythme (Phares, 1971 ; Borchert, 1975 ; Farmer, 1975 ; Champagnat et al, 1986 ; Dupré et al, 1986 ; Crabbé, 1987 ; Millet et al, 1991 ; Parmentier et Barnola, 1993)

Ces rythmes permettent notamment de protéger l'arbre contre des conditions momentanément défavorables (sécheresse estivale par exemple), pendant lesquelles l'allongement est stoppé et le méristème apical protégé par un bourgeon ; l'arbre peut ensuite profiter de conditions à nouveau favorables pour développer une nouvelle vague de croissance et prolonger ainsi son développement annuel. Cette irrégularité de croissance peut avoir des conséquences sur la structure de la plante (Collin et al, 1993 ; Falcone et al, 1986). Elle s'accompagne en effet souvent d'une hétérogénéité structurale par le fait que chaque variation du rythme se traduit par une modification morphologique plus ou moins marquée (hétéroblastie de l'unité de croissance, dimensions et forme des feuilles, longueur des entre-nœuds, diamètre de la moelle, angle entre les unités successives : Édelin, 1993). Ces modifications peuvent avoir des conséquences plus ou moins favorables sur la qualité du bois (Guard et Postlethwait, sd ; Igboanugo, 1990 ; Parmentier et al, 1991). La croissance en épaisseur peut également être affectée (Igboanugo, 1990).

Parmi les essences ligneuses tempérées, les chênes présentent presque tous des rythmes très apparentés : en conditions naturelles, ils peuvent montrer de 1 vague (monocyclisme) à 2, voire 3-4 vagues (polycyclisme) de croissance séparées par des périodes de repos nettes relativement courtes (de 1 à quelques semaines). Dans les régions tempérées, la première vague a lieu au printemps, et les suivantes dans le cours de l'été. Cela peut leur permettre, selon les conditions extérieures, de prolonger leur allongement jusqu'en septembre ou au contraire de le limiter à 2 sem en avril pour la totalité de l'année. C'est le cas du chêne rouge d'Amérique (Meredieu, 1993). Mais si l'on connaît de façon précise chez les chênes les caractéristiques du rythme et ses fluctuations en conditions contrôlées (Farmer, 1975 ; Champagnat et al, 1986 ; Collin et al, 1993 ; Parmentier et Barnola, 1993), on connaît peu ses manifestations en conditions naturelles où sont souvent décrites seulement les variations interindividuelles ou selon l'âge, mais très rarement selon les facteurs physiques du milieu (Reich et al, 1980 ; Igboanugo, 1990 ; Meredieu, 1993).

La présente étude se situe dans le cadre d'une expérimentation de terrain destinée à évaluer l'influence de la fertilisation, du désherbage et des microvariations d'un sol d'origine agricole sur la croissance et la ramification initiales de jeunes plants de chêne rouge d'Amérique. Dès la première année de croissance (1992), certains plants ont exprimé un développement polycyclique d'une manière non-aléatoire, tandis que les autres n'ont formé qu'un seul cycle. L'ob- 
jectif de cet article est de mettre en évidence quels ont été les facteurs du milieu qui ont permis ce type de développement, et d'évaluer les conséquences de celui-ci sur l'installation d'une plantation de chêne rouge. En effet, le développement polycyclique aboutit, chez beaucoup d'espèces (Immel, 1978; Kremer, 1981) à une augmentation substantielle de la croissance, et pourrait donc contribuer notablement, aux stades juvéniles, à la réussite d'une plantation. La connaissance des facteurs favorisants pourrait ainsi permettre une meilleure maîtrise dans la mise en place des plantations de chêne rouge d'Amérique, même si le polycyclisme aboutit également à une ramification plus abondante pouvant induire des défauts de forme durables au stade adulte (Dupré et al, 1986).

\section{MATÉRIEL ET MÉTHODES}

\section{Site, protocole et plan expérimental}

Les données sont issues d'un essai de terrain installé début 1992 dans le sud-ouest de la France (Gers), aux coordonnées $43^{\circ} 52^{\prime}$ de latitude nord et $0^{\circ} 0^{\prime}$ de longitude par rapport au méridien international. La parcelle, d'origine agricole (maïs), a une surface de 2,5 hectares et présente un micro-relief (fig 1 ).

Le climat moyen des années 1983-1991 montre des précipitations annuelles comprises entre 800 et $900 \mathrm{~mm}$, bien réparties sur tous les mois ; une température moyenne annuelle de $13,5^{\circ} \mathrm{C}$ (mini mensuel moyen en janvier : $3,7^{\circ} \mathrm{C}$; maxi mensuel moyen en juillet : $21,5^{\circ} \mathrm{C}$ ). L'année 1992, durant laquelle les observations ont été faites, a eu pour particularité de fortes précipitations en juin ( 220 au lieu de $75 \mathrm{~mm}$ ) et en octobre (200 au lieu de $65 \mathrm{~mm}$ ).

Le sol est de type lessivé dégradé plus ou moins hydromorphe (Duchaufour, 1970); c'est une "boulbène" selon la dénomination vernaculaire. La texture varie de limono-sableuse en surface à limono-argileuse en profondeur. II existe des traces (taches rouilles) d'une nappe perchée temporaire dont la profondeur varie de $35 \mathrm{~cm}$ dans les parties basses du bloc 1, le plus humide, à $1 \mathrm{~m}$ dans les parties hautes du bloc 2 , le plus $\mathrm{sec}$, avec la présence en plancher d'un horizon Bt à structure massive, très compact.

On a utilisé des plants de chêne rouge de type «1-0» de hauteur moyenne $20 \mathrm{~cm}$, dont les glands sont issus d'un seul peuplement classé du sudouest (Nabas, Pyrénées-Atlantiques). Les plants étaient à racine nue et leurs racines ont été habillées et prâlinées à la plantation.

Trois facteurs ont été testés, selon plusieurs modalités entièrement croisées entre elles : fertilisation (oui/non), désherbage (oui/non) et écartement entre les plants $(1,2$ et $4 \mathrm{~m}$ ). La fertilisation a été appliquée en plein à la plantation, par épandage en surface de 3 types de granulés : ammonitrate, soit $60 \mathrm{U} / \mathrm{ha}$ d'azote, superphosphate triple, soit $100 \mathrm{U} / \mathrm{ha}$ de phosphore, et chlorure de potassium, soit $50 \mathrm{U} / \mathrm{ha}$ de potassium. Le tapis herbacé a été totalement éliminé chimiquement en modalité désherbée par application en plein répétée en cours de saison de glyphosate (marque commerciale "round up") à raison de $1800 \mathrm{~g} / \mathrm{ha}$ de matière active.

Le dispositif est organisé en «blocs aléatoires incomplets» (Dagnélie, 1984). II comprend 3 blocs qui correspondent à 3 niveaux différents d'humidité du sol. Ainsi, en fin d'été, les pF mesurés à $20 \mathrm{~cm}$ de profondeur à l'aide de sondes à plâtre s'échelonnent par exemple entre 2,4 (bloc 1), 2,52,6 (bloc 3) et 2,7 (bloc 2). Dans les blocs, les combinaisons de modalités de facteurs sont appliquées à des placeaux unitaires de 30 plants. Chaque placeau est entouré d'une zone d'au moins $5 \mathrm{~m}$ où la même densité de plantation et les mêmes modalités de désherbage/fertilisation sont appliquées (fig 2).

\section{Mesures et échantillonnage}

Sur la totalité des plants des placeaux expérimentaux, on a mesuré le diamètre à $8 \mathrm{~cm}$ du sol (repère matérialisé au feutre), au millimètre près, et la hauteur totale (hauteur verticale de la pousse la plus haute), au cm près. Ces mesures ont été effectuées avant et après la période de croissance totale de l'année 1992. De plus, l'apparition ou non de pousses supplémentaires d'été (polycyclisme) a été notée pour chaque plant lors d'observations hebdomadaires effectuées à partir de début mai 1992. 
L'estimation de la surface foliaire par plant a porté sur 22 placeaux (tableau I). Elle a été effectuée sur tous les plants en fin de croissance selon les 2 étapes suivantes : i) comptage du nombre de feuilles $(n i)$ dans chacune des 3 catégories de longueur : $<10 \mathrm{~cm}, 10-15 \mathrm{~cm},>15 \mathrm{~cm}$, en distinguant les feuilles de la première vague de croissance (feuilles de printemps) des feuilles des vagues suivantes (feuilles d'été) ; ii) détermination au curvimètre de la surface individuelle d'une cinquantaine de feuilles par catégorie prélevées au hasard sur les 22 placeaux, dont on a déduit la surface moyenne par catégorie (si), d'où la surface totale par plant par sommation des produits ni*si.

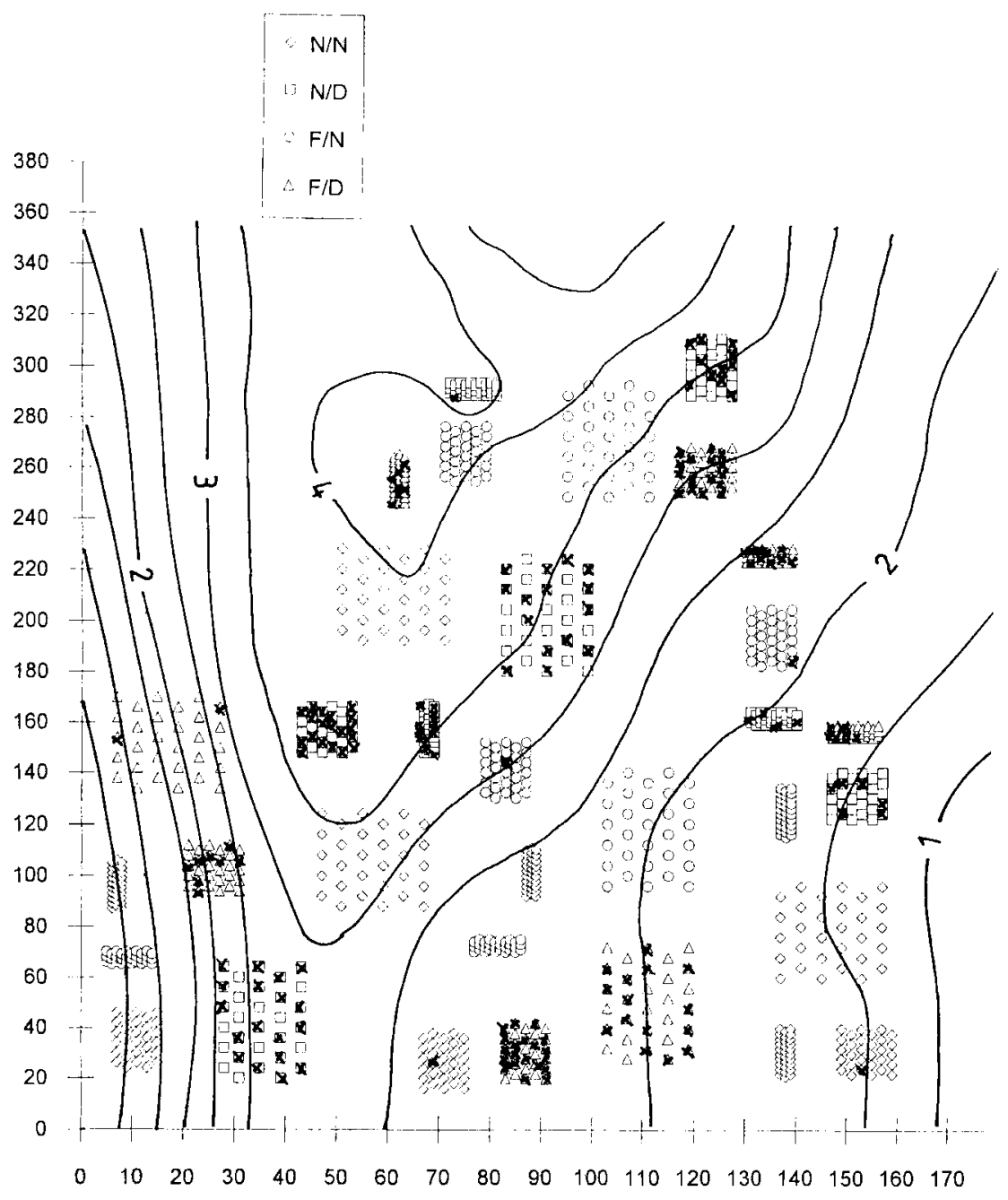

Fig 1. Plan du dispositif expérimental. Les symboles représentent les plants, la croix en surcharge correspond aux plants polycycliques en 1992. Le microrelief est caractérisé par les lignes d'égale altitude équidistantes de $0,5 \mathrm{~m}$. Les coordonnées des axes sont exprimées en mètre sur l'abscisse et en demi-mètre sur l'ordonnée. 


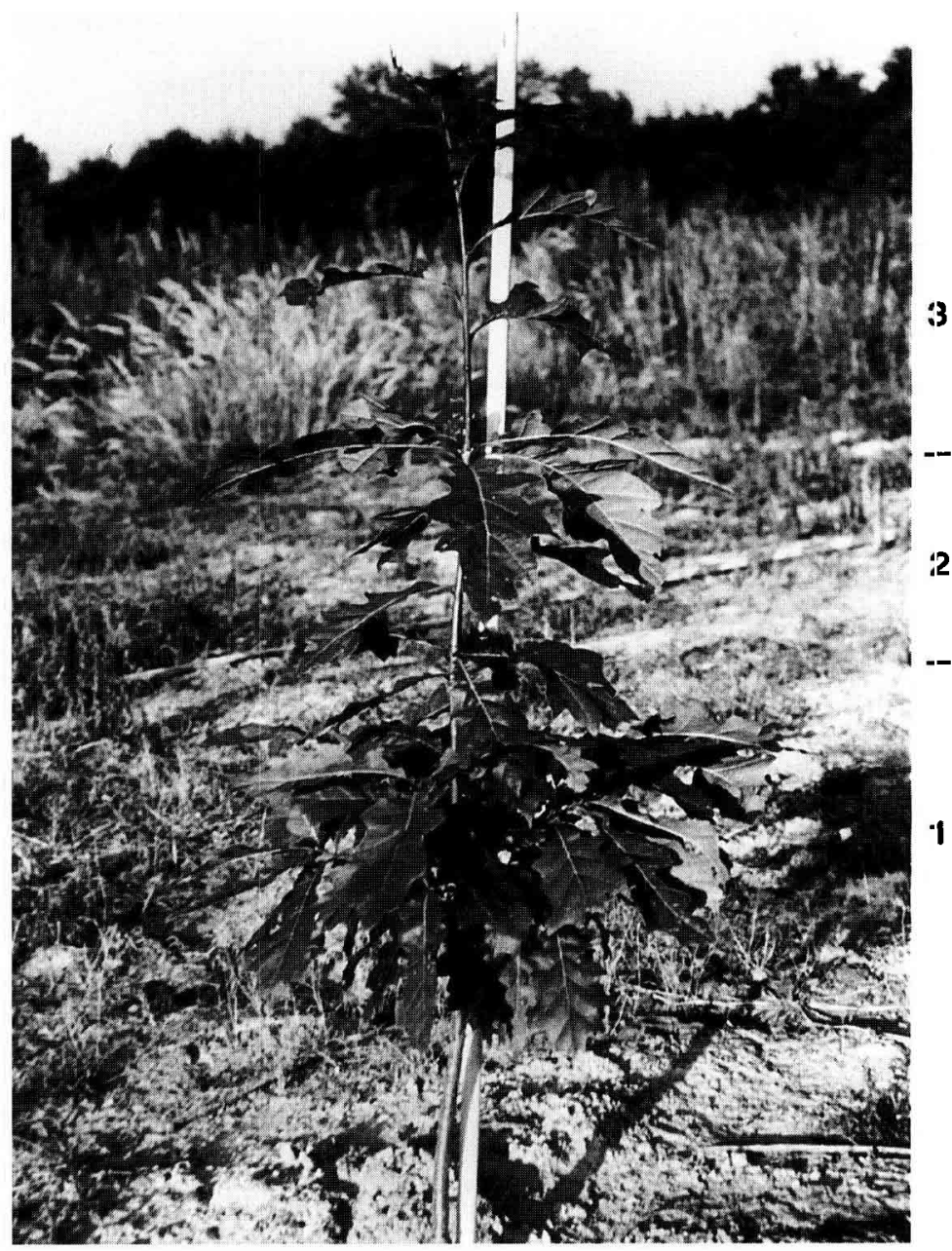

Fig 2. Plant de chêne rouge polycyclique présentant 3 cycles d'allongement successits (le troisième est en cours de croissance). Noter la plus grande dimension des feuilles situées au sommet du deuxième cycle.

Un suivi annuel fin du développement de la totalité de l'appareil aérien a été par ailleurs réalisé sur un échantillon de 300 plants répartis dans 10 placeaux ( $c f$ tableau I). Le protocole adopté s'inspire de celui décrit par Barthélemy et Caraglio, 1991 : sur chaque plant, on a repéré les différentes unités de croissance infra-annuelles en distinguant l'axe principal (le plus haut) des ramifications. Chaque unité de croissance a été mesurée en longueur (au millimètre près) et en diamètre en son milieu (au 1/10 mm près).

\section{Analyse des données}

Les données ont été analysées graphiquement et analytiquement (analyses de variance, tableaux de contingence et $\chi^{2}$ à l'aide du logiciel "S" (Chambers et Hastie, 1992). Les tests de comparaison de pourcentage ont été effectués par la méthode décrite dans Heller (1968). Les tests non-paramétriques sont issus de Sprent (1993) : test de Wilcoxon pour 2 échantillons, et test de Kruskal-Wallis pour plus de 2 échantillons. 
Tableau I. Répartition par bloc et par traitement des placeaux échantillons utilisés pour l'étude de l'architecture et de l'appareil foliaire.

\section{Bloc Écartement Fertilisation Désherbage Échantillon architecture Échantillon feuilles}

\begin{tabular}{|c|c|c|c|}
\hline $1 \times 1 \mathrm{~m}$ & oui & oui & \\
\hline $1 \times 1 \mathrm{~m}$ & oui & non & \\
\hline $1 \times 1 \mathrm{~m}$ & non & oui & \\
\hline $1 \times 1 \mathrm{~m}$ & non & non & \\
\hline $2 \times 2 \mathrm{~m}$ & oui & oui & \\
\hline $2 \times 2 \mathrm{~m}$ & oui & non & \\
\hline $2 \times 2 \mathrm{~m}$ & non & oui & $x$ \\
\hline $2 \times 2 \mathrm{~m}$ & non & non & $\mathrm{x}$ \\
\hline $4 \times 4 m$ & oui & oui & \\
\hline $4 \times 4 m$ & non & oui & \\
\hline $4 \times 4 \mathrm{~m}$ & non & non & \\
\hline
\end{tabular}

\begin{tabular}{|c|c|c|c|c|c|}
\hline 2 & $1 \times 1 \mathrm{~m}$ & oui & oui & & $x$ \\
\hline 2 & $1 \times 1 \mathrm{~m}$ & oui & non & & \\
\hline 2 & $1 \times 1 \mathrm{~m}$ & non & oui & & $x$ \\
\hline 2 & $1 \times 1 \mathrm{~m}$ & non & non & & \\
\hline 2 & $2 \times 2 \mathrm{~m}$ & oui & oui & $x$ & $x$ \\
\hline 2 & $2 \times 2 m$ & oui & non & $x$ & $x$ \\
\hline 2 & $2 \times 2 \mathrm{~m}$ & non & oui & $x$ & $x$ \\
\hline 2 & $2 \times 2 m$ & non & non & $x$ & $x$ \\
\hline 2 & $4 \times 4 m$ & oui & non & & \\
\hline 2 & $4 \times 4 \mathrm{~m}$ & non & oui & & $x$ \\
\hline 2 & $4 \times 4 \mathrm{~m}$ & non & non & & \\
\hline 3 & $1 \times 1 \mathrm{~m}$ & oui & oui & & $x$ \\
\hline 3 & $1 \times 1 \mathrm{~m}$ & oui & non & & \\
\hline 3 & $1 \times 1 \mathrm{~m}$ & non & oui & & $x$ \\
\hline 3 & $1 \times 1 \mathrm{~m}$ & non & non & & \\
\hline 3 & $2 \times 2 m$ & oui & oui & $x$ & $x$ \\
\hline 3 & $2 \times 2 m$ & oui & non & $x$ & $x$ \\
\hline 3 & $2 \times 2 m$ & non & oui & $x$ & $x$ \\
\hline 3 & $2 \times 2 \mathrm{~m}$ & non & non & $x$ & $x$ \\
\hline 3 & $4 \times 4 m$ & oui & oui & & $x$ \\
\hline 3 & $4 \times 4 \mathrm{~m}$ & oui & non & & \\
\hline 3 & $4 \times 4 \mathrm{~m}$ & non & non & & \\
\hline
\end{tabular}




\section{RÉSULTATS}

À noter que la mortalité globale des plants n'a pas dépassé 1\% en 1992.

\section{Déterminisme du polycyclisme}

\section{Effet des facteurs contrôlés sur le taux de plants polycycliques par placeau}

En 1992, une première vague de croissance est apparue en avril. À partir de fin mai et jusqu'en septembre, certains plants ont développé de 1 à 2 cycles supplémentaires morphologiquement bien caractérisés (fig 2).

La figure 3 montre la répartition du pourcentage de plants polycycliques par placeau. On voit que cette proportion varie entre 0 et plus de $60 \%$ des plants. Le fait le plus frappant est la disjonction nette entre le groupe des placeaux désherbés (qui contient 279 plants polycycliques), et celui des placeaux enherbés (qui contient seulement 4 plants polycycliques) : l'influence positive du désherbage sur le taux de plants polycycliques est donc hautement significative, et la figure 3 montre que cela reste vrai dans chaque bloc.
On constate néanmoins sur la figure 3 que cette influence du désherbage semble dépendante du bloc. Un test de $\chi^{2}$ portant sur les seuls placeaux désherbés met en effet en évidence que les blocs sont globalement hétérogènes entre eux à ce point de vue $\left(\chi^{2}=15,9\right)$; la comparaison des pourcentages conclut à une différence hautement significative entre le bloc 2 et les blocs 1 et 3 (valeurs respectives de $t: 3,7$ et 2,9 ), et à l'absence de différence significative entre les blocs 1 et $3(t=0,6)$ : le bloc 2 contient donc une quantité significativement plus élevée de plants polycycliques. Une analyse spatiale plus fine du polycyclisme basée sur la figure 1 fait apparaître une liaison avec le microrelief : on note une plus grande densité de plants polycycliques sur les zones de plus grande pente du bloc 2 .

Globalement, la fertilisation ne semble pas introduire d'hétérogénéité dans la distribution des plants polycycliques entre les différents placeaux, aussi bien globalement $\left(\chi^{2}=0,03\right)$ qu'à l'intérieur de chaque modalité de désherbage $\left(\chi^{2}=0,08\right.$ pour le cas désherbé, et $\chi^{2}=0,012$ pour la modalité non-désherbée). Toutefois, dans le détail, le facteur fertilisation associé à la modalité désherbée introduit une hétérogénéité significative dans les blocs $1\left(\chi^{2}=11,8\right)$ et 3

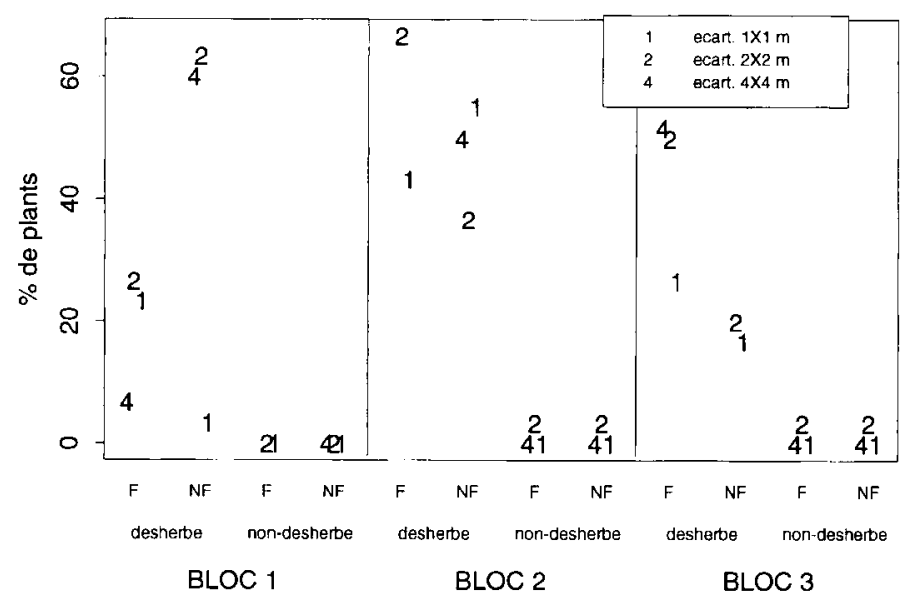

BLOC
BLOC 2
Fig 3. Positionnement relatif des placeaux du dispositif par leur taux de plants polycycliques en 1992. Chaque chiffre représente un placeau. 
$\left(\chi^{2}=9,6\right)$; la comparaison des pourcentages montre que cette hétérogénéité pourrait correspondre à un effet de la fertilisation négatif dans le cas du bloc $1(t=2,7)$ et positif dans le cas du bloc $3(t=3,1)$. La densité de plantation est un facteur d'hétérogénéité de la distribution du polycyclisme dans le seul bloc $1\left(\chi^{2}=13,8\right)$ : cette hétérogénéité est due aux placeaux d'écartement $1 \mathrm{~m}$ qui, dans ce bloc, contiennent significativement moins de plants polycycliques.

Le polycyclisme s'étant presque exclusivement exprimé en modalité désherbée, et aussi par souci d'homogénéité des données, les résultats suivants portent uniquement sur des plants des modalités désherbées.

\section{Effet des facteurs contrôlés sur le degré de polycyclisme par plant}

L'étude a porté sur les seuls plants polycycliques de l'échantillon de 300 plants mesurés en détail. Le degré de polycyclisme est apprécié par le "volume d'été» par plant, qui correspond à la somme des volumes de bois de toutes les pousses d'été du plant, le volume étant estimé par la formule $v=$ $3,1416^{\star} d^{2 \star} / / 4$ où $d$ est le diamètre au milieu de la pousse et / sa longueur.
La répartition de ces volumes dans les différents traitements concernés par l'échantillon est indiquée dans le tableau II. Du fait de la non-normalité des distributions, des faibles effectifs de plants présentant un grand volume ( $c f$ «Relation entre pourcentage de plants polycycliques et importance du polycyclisme par plant») et de l'hétérogénéité des variances, les comparaisons entre traitements ont été effectuées à l'aide de tests non-paramétriques. La comparaison des blocs en modalité non-fertilisée (test de Kruskal-Wallis) donne un $\chi^{2}$ de 4,84 , ce qui indique une absence d'hétérogénéité. En revanche, la confrontation des modalités fertilisé et non-fertilisé (test de Wolcoxon) des blocs 2 et 3 indique une différence significative (variable gaussienne égale à 2,5), ce qui indiquerait une influence positive de la fertilisation sur le développement estival des plants dans ces 2 blocs.

\section{Effet des dimensions initiales du plant sur la probabilité de polycyclisme}

On a pris en compte ici le diamètre et la hauteur initiaux des plants. La figure 4 montre clairement que les plants polycycliques ont des diamètres initiaux significativement plus faibles que ceux des plants monocycliques (non-recouvrement des inter-

Tableau II. Répartition de la moyenne du volume de bois de pousse d'été par plant polycyclique $\left(\mathrm{cm}^{3}\right)$, de sa variabilité $\left(\mathrm{cm}^{3}\right)$, et de l'effectif de plants polycycliques selon les blocs et les modalités de fertilisation.

\begin{tabular}{|c|c|c|c|c|c|c|c|}
\hline \multirow{2}{*}{\multicolumn{2}{|c|}{$\begin{array}{r}\text { Bloc } 1 \\
\text { Non-fertilisé }\end{array}$}} & \multicolumn{3}{|c|}{ Bloc 2} & \multicolumn{3}{|c|}{ Bloc 3} \\
\hline & & Fertilisé & Non-fertilisé & Total & Fertilisé & Non-fertilisé & Total \\
\hline & & & 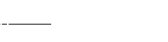 & - & & -- & \\
\hline & 2,6 & 5,3 & 1,6 & 4, & 2,7 & 0,5 & 2,1 \\
\hline Écart type & 3,6 & 6,0 & 2,8 & 5,4 & 4,9 & 0,5 & 4,2 \\
\hline Effectif & 20 & 19 & 10 & 29 & 15 & 6 & 21 \\
\hline
\end{tabular}

Ces calculs concernent les 300 plants de l'échantillon "architecture" (cf tableau I). 

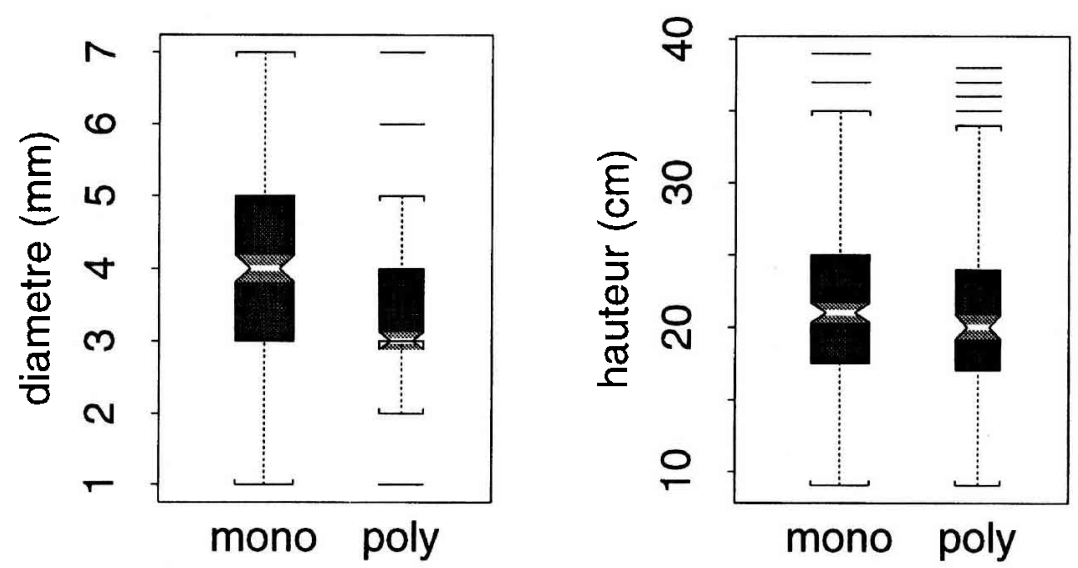

Fig 4. Distributions des diamètres et hauteurs initiaux des 2 catégories de plants (monocycliques et polycycliques). Dans cette représentation en «boxplot», la boîte contient verticalement la moitié centrale de la distribution ( $50 \%$ des plants autour de la médiane), la médiane est symbolisée par le trait blanc située dans la boîte, et son intervalle de confiance par les 2 encoches latérales ; les pointillés verticaux rejoignent les points individualisés situés à moins de 1,5 fois l'interquartile de la distribution ; les valeurs extérieures sont représentées par des traits horizontaux ; enfin, la largeur de la boîte est proportionnelle à la racine carrée de l'effectif de la distribution.

vailes de confiance des médianes). De plus, la distribution de leurs diamètres est fortement dissymétrique. En revanche, on voit que les hauteurs initiales sont comparables chez les 2 types de plants.

\section{Influence des dimensions initiales du plant sur son degré de polycyclisme}

Les données analysées sont issues des seuls plants polycycliques de l'échantillon. L'hypothèse d'une dépendance entre les dimensions initiales des plants et l'importance du polycyclisme par plant (mesurée par le volume ligneux formé par les vagues de croissance estivales) a été testée par un test de $\chi^{2}$. Cette hypothèse est rejetée au risque de $5 \%$, tant pour le diamètre que pour la hauteur.

En revanche, le tableau III montre que le volume d'été par plant, s'il n'est pas lié à la catégorie de dimension initiale du plant, augmente toutefois avec l'effectif total de plants appartenant à cette catégorie de dimensions. Ce phénomène s'apparente à l'occurrence aléatoire d'événéments rares (ici l'existence de plants à grand volume d'été), dont la probabilité d'apparition est relativement constante, comme dans les processus poissonniens.

\section{Relation entre pourcentage de plants polycycliques et importance du polycyclisme par plant}

On est ici à l'échelle du placeau. La figure 5 montre une relation croissante sublinéaire entre le pourcentage de plants polycycliques par placeau et le volume moyen d'été par plant de ce placeau. Cette dépendance entre les 2 phénomènes est à rapprocher des résultats du paragraphe précédent : il le généralise à l'échelle du placeau quelle que soit la classe de dimension, et a sans doute la même origine (faible probabilité constante des fortes croissances estivales). 


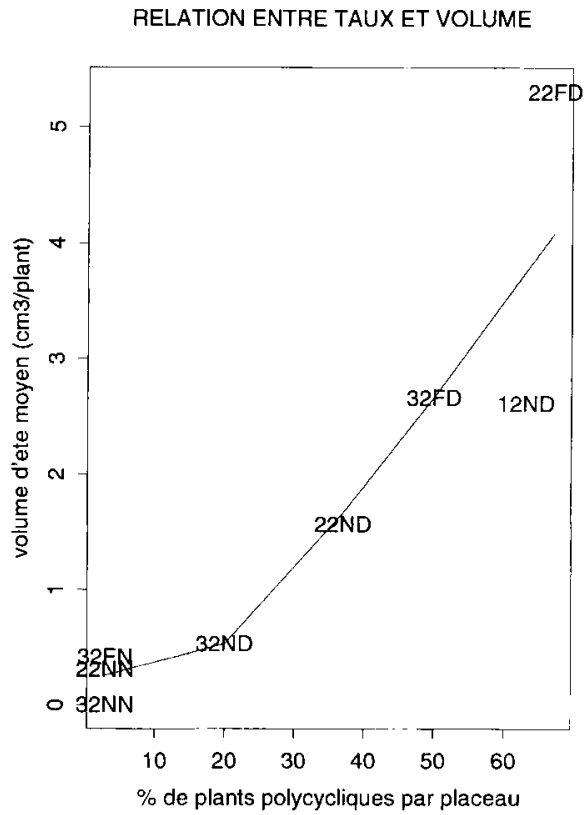

Fig 5. Relation entre le volume d'été moyen par plant polycyclique au niveau d'un placeau et le pourcentage de plants polycycliques de ce placeau. Le code à 4 caractères représentant un placeau contient successivement : le numéro du bloc $(1,2$ ou 3$)$, l'écartement $(1,2$ ou 4$)$, la fertilisation ( $N$ pour non, $F$ pour oui) et le désherbage ( $N$ pour non, $D$ pour oui).

\section{Conséquences du polycyclisme}

\section{Sur l'axe principal (diamètre et hauteur totale)}

La répartition des accroissements annuels pour 1992 selon les modalités est représentée sur la figure 6 . On y voit notamment un effet marqué du polycyclisme sur la croissance en diamètre, particulièrement dans les blocs 1 et 2, qui aboutit à plus que doubler l'accroissement, lequel passe globalement de 0,99 à $2,3 \mathrm{~mm}$. Les 2 variables ayant une distribution approximativement gausienne, il a été possible de les traiter par analyse de variance. Nous avons utilisé le modèle suivant au niveau arbre :
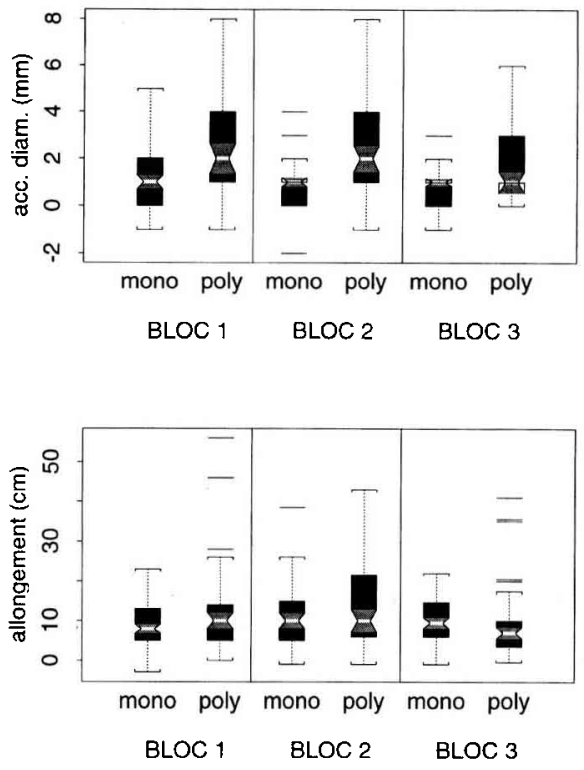

Fig 6. Conséquences du polycyclisme sur la croissance en diamètre et en hauteur des plants. Répartition par bloc cf figure 4 pour le mode de représentation.

acdt ou acht

$=b l o c+p o l y c+$ écart + fert

$+b^{*} / c^{*} p o l y c+$ écart ${ }^{*}$ polyc + fert $^{*}$ polyc

où acdt et acht sont respectivement les accroissements annuels en diamètre et en hauteur ; bloc représente le facteur bloc ; polyc, le facteur polycyclisme ; écart, l'écartement entre les plants ; et fert, le facteur fertilisation.

Le facteur polycyclisme a un effet global sur l'accroissement en diamètre qui est hautement significatif ( $p=0,006)$, et n'interagit pas avec le bloc, la fertilisation ou l'écartement (respectivement : $p=0,25 ; p=0,34$; $p=0,07$ ).

En revanche, le polycyclisme n'a pas d'effet significatif net sur l'accroissement en hauteur $(p=0,10)$, et n'intervient pas non plus indirectement sous forme d'interaction significative avec les autres facteurs $(p=$ 
Tableau III. Relation entre les distributions des plants dans chaque catégorie de diamètre et de hauteur initiaux et les effectifs de plants par catégorie. On a donné une limite arbitraire de volume $\left(5000 \mathrm{~cm}^{3}\right)$ et les interquartiles extrêmes pour caractériser les parties droite et gauche des distributions.

Catégorie de diamètre $(\mathrm{mm})$

Effectif total

Nb plants de volume $<5000 \mathrm{~cm}^{3}$

$\mathrm{Nb}$ plants volume $>5000 \mathrm{~cm}^{3}$

1 er interquartile

$4{ }^{\ominus}$ interquartile

Catégorie de hauteur $(\mathrm{cm})$

Effectif total

$\mathrm{Nb}$ plants de volume $<5000 \mathrm{~cm}^{3}$

$\mathrm{Nb}$ plants de volume $>5000 \mathrm{~cm}^{3}$

1er interquartile

$4^{e}$ interquartile
$<15$

$15-20$

$$
2
$$

$\begin{array}{rr}4 & 40 \\ 4 & 32 \\ 0 & 8 \\ 109 & 400\end{array}$

$41 \quad 15345$

$\begin{array}{rr}9 & 2 \\ 9 & 18 \\ 0 & \\ 120 & 483 \\ 332 & 14084\end{array}$

3

40

32

8
4

5

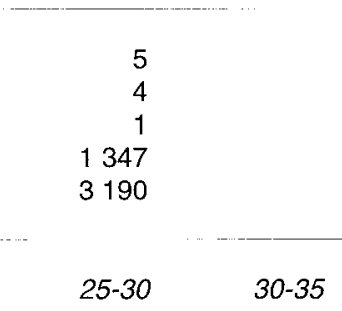

0,08 pour le bloc ; $p=0,70$ pour la fertilisation ; $p=0,50$ pour l'écartement).

\section{Sur l'ensemble du plant}

\section{Appareil foliaire}

La figure 7 montre que le nombre total de feuilles par plant est toujours supérieur chez les plants polycycliques, quelle que soit la dimension initiale du plant. Toutefois, ce nombre augmentant légèrement avec la dimension du plant monocyclique, alors qu'il est relativement constant chez les plants polycycliques, les gros plants monocycliques ont un nombre total de feuilles équivalent à celui des polycycliques de même dimension. Dans le détail, on s'aperçoit que les plants polycycliques ont un supplément estival de feuilles qui s'ajoute de manière homogène aux feuilles de printemps, indépendamment de la dimension du plant. En définitive, le nombre total de feuilles par plant est en moyenne de 21,3 chez les poly- cycliques et de 12,6 chez les monocycliques. L'analyse du tableau de contingence basé sur 3 classes de nombre de feuilles confirme ( $\chi^{2}$ observé de 90 ) que cette différence est hautement significative.

On voit sur la figure 8 que les plants polycycliques ont, au printemps, des feuilles de cycle 1 dont la surface moyenne est analogue à celle des plants monocycliques $\left(27 \mathrm{~cm}^{2}\right)$ et qu'ensuite se mettent en place des feuilles d'été de surface unitaire beaucoup plus grande $\left(44 \mathrm{~cm}^{2}\right)$.

Au total, les plants monocycliques ont une surface foliaire de $330 \mathrm{~cm}^{2}$ en moyenne, contre $750 \mathrm{~cm}^{2}$ pour les plants polycycliques.

\section{Appareil ligneux aérien}

La figure 9 permet de comparer les volumes ligneux aériens des 2 types de plants : on voit que les plants polycycliques ont un volume aérien 4 fois supérieur, et aussi beaucoup plus variable que les plants monocy- 

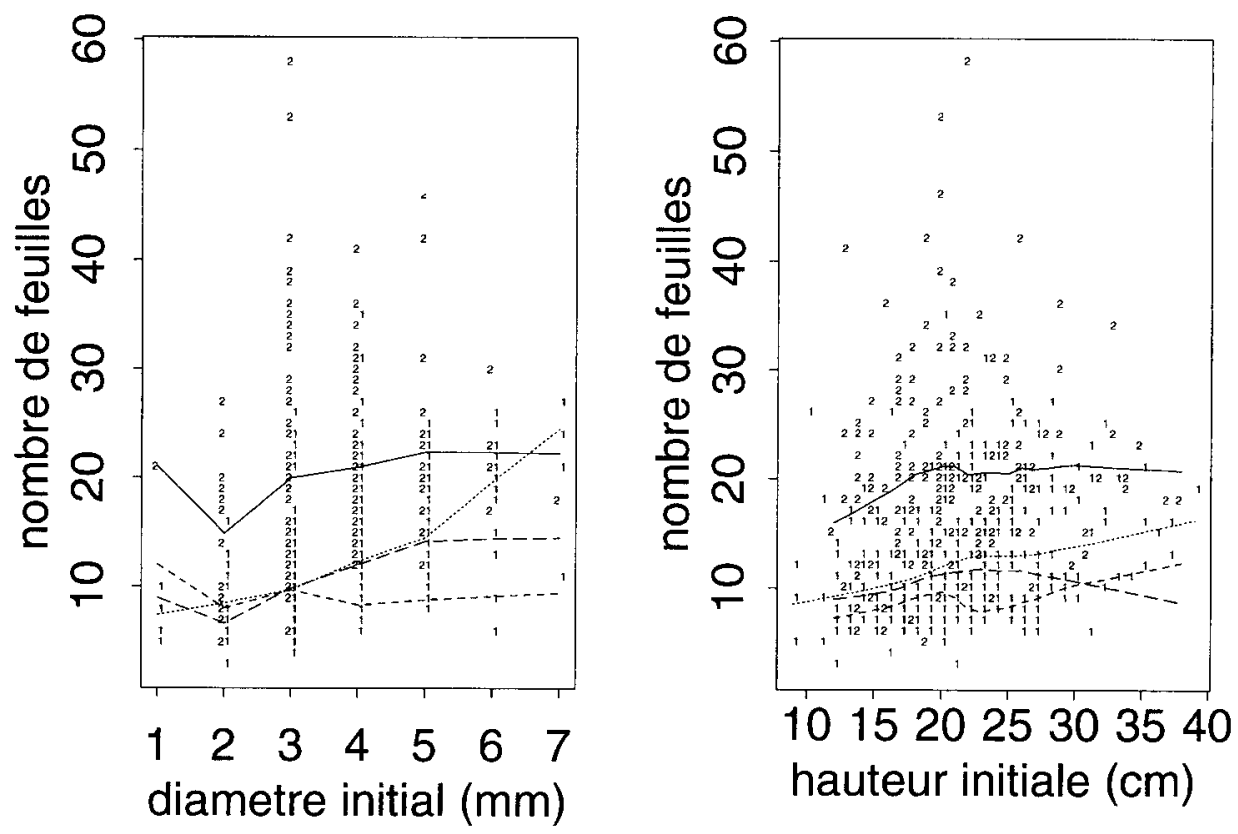

Fig 7. Relations entre le nombre de feuilles par plant et la dimension initiale du plant selon le type de plant et le type de feuille. 1 mono ; $2 \longrightarrow$ poly-total ; 2 poly-ete ; 2 - - - - poly-print.

cliques. La différence reste néanmoins significative (non-recouvrement des intervalles de confiance des médianes). Cette différence s'explique par le caractère «supplémentaire» du volume de pousses d'été $\left(3 \mathrm{~cm}^{3}\right)$, car les volumes (ainsi que les sommes de longueurs) sont équivalents au printemps chez les 2 types de plants $\left(\chi^{2}\right.$ de 1,84 avec 3 classes de volume). Par ailleurs, les unités de croissance estivales font doubler la somme de longueur de pousses par plant, qui passe de 16 à $34,5 \mathrm{~cm}$ en moyenne.

La prise en compte de l'appartenance ou non de chaque pousse à l'axe principal du plant nous a par ailleurs permis d'étudier les variations du taux de ramification

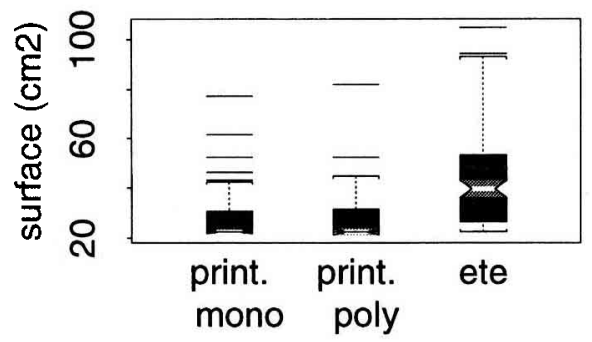

Fig 8. Comparaison des surfaces movennes par feuille selon le type de plant et la vague de croissance.

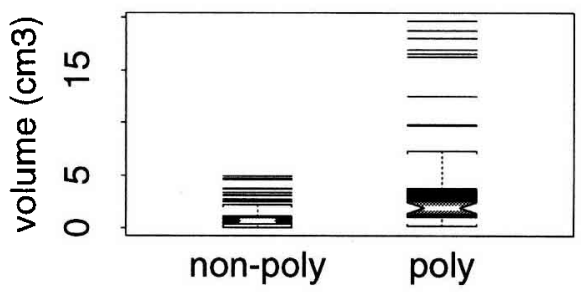

Fig 9. Comparaison des distributions de volume ligneux aérien par plant pour les plants monocycliques et les plants polycycliques. 
des plants. Le caractère polycyclique fait passer le taux de ramification en volume de 24 à $61 \%$ en moyenne. Cette différence est hautement significative sur le plan statistique $\left(\chi^{2}\right.$ de 63 avec 3 classes de taux, et taux de répartition des 2 types de plants dans les classes extrêmes significativement différent : $t=4,6$ ). En ne prenant en compte que les plants qui n'ont pas eu d'avortement de bourgeon terminal d'axe principal début 1992 , et qui ne sont donc pas déjà ramifiés initialement, le taux passe de 12 à $63 \%$. En revanche, les taux de ramification des 2 types de plants au printemps ne diffèrent pas significativement entre eux $\left(\chi^{2}\right.$ de 5,1 pour les taux respectifs de 24 et $35 \%$ ). Les mêmes phénomènes sont notés pour le taux de ramification exprimé par les sommes de longueur de pousses: le taux passe de 28 à $63 \%$, et il n'est pas significativement différent pour les seules pousses de printemps (29 et $41 \%$ ). Si l'on compare maintenant, chez les seuls plants polycycliques, le taux de ramification volumique de la vague de croissance 1 (printemps), qui est de $35 \%$, avec celui des vagues estivales, qui est de $79 \%$, on voit que le développement estival se porte proportionnellement davantage sur la ramification que le développement printanier (en toute rigueur, le "volume de printemps» utilisé ici contient l'épaississement annuel ; mais ce résultat reste a fortiori valable pour le volume de printemps stricto sensu, puisque l'épaississement estival de l'axe principal est plutôt supérieur à celui des ramifications).

\section{DISCUSSION}

\section{Facteurs du polycyclisme}

Nous avons vu que le principal facteur contrôlé actif sur la probabilité de développement polycyciique des plants est ici le désherbage. Cette influence est modulée selon le bloc (fig 3), donc a priori selon le niveau moyen d'humidité. Mais l'absence de tapis herbacé entraîne à elle seule la modification de nombreux paramètres de l'environnement immédiat des plants, car lors de l'apparition de la deuxième vague de croissance, fin juin, les herbacées des placeaux non-désherbés dépassaient en hauteur la plupart des plants (Meredieu, 1993) : le désherbage aboutit donc à la modification des conditions de lumière, de température, d'humidité et de concentration en gaz carbonique de l'air, ainsi qu'à une absence de compétition au niveau racinaire à laquelle les jeunes plants peuvent être sensibles jusqu'à un rayon de $30 \mathrm{~cm}$ (Smethurst et al, 1993) et entrer de ce fait en repos (Farmer, 1975). La littérature fait état de l'influence positive, dans certaines conditions souvent contrôlées, de l'intensité de la lumière (Igboanugo, 1990 ; Millet et al, 1991 ; Reich et al, 1980), ou de sa durée (Collin et al, 1993) sur le nombre de cycles de jeunes arbres ; il en est de même pour la température (Farmer, 1975 ; Millet et al, 1991). En revanche, peu de références directes existent pour l'influence de l'humidité du sol : Millet et al (1991) indiquent que les pluies peuvent assurer le déclenchement synchrone d'un cycle ; Smethurst et al (1993) montrent que la présence d'herbacées ralentit l'absorption racinaire de jeunes arbres. En ce qui concerne la répartition spatiale des plants polycycliques en relation avec le microrelief (fig 1 ), on peut formuler l'hypothèse suivante : compte tenu de la forte pluviométrie du mois de juin 1992 précédant la deuxième vague de croissance (cf Matériel et méthodes), il pourrait y avoir eu un effet favorable du drainage sur pente, l'engorgement plus durable des autres zones n'ayant pas permis l'expression du polycyclisme. Cette hypothèse d'une relation étroite entre activité racinaire et croissance aérienne est étayée par l'existence d'une croissance rythmique également au niveau racinaire chez certains ligneux (Le Roux et al, 1993). 
L'absence d'influence de la fertilisation sur le polycyclisme (en modalité désherbée) rejoint les observations faites par Immel et al (1978) et Kleiner et al (1992) sur les relations entre fertilisation et croissance chez de jeunes chênes rouges lorsque les autres facteurs du milieu sont favorables. En revanche, Phares (1971) a montré que la fertilisation pouvait favoriser le polycyclisme en conditions de lumière limitante, et Dupré et al (1986) signalent le fort déterminisme du polycyclisme par rapport à la fertilité du sol. Le problème est sans doute complexe si I'on considère, comme Parmentier et Barnola (1993), que la nature chimique des produits apportés peut intervenir dans la réponse rythmique du plant. Dans notre cas, outre la qualité de l'apport minéral, on peut penser que cet ancien sol agricole, relativement riche dans les premiers centimètres $\left(0,03 \%\right.$ de $\mathrm{P}_{2} 05,0,3 \mathrm{meq} / 100$ de $\mathrm{Mg}, 0,2$ meq/100 de K, 3,2 meq/100 de $\mathrm{Ca}$ ), n'a pas représenté un facteur limitant du polycyclisme au point de vue minéral, tout au moins dans les conditions de l'étude (compétition racinaire nulle entre les plants désherbés). De plus, la fertilisation a pu n'avoir qu'un effet très minime compte tenu de la reconstitution partielle de l'appareil racinaire et de son cortège mycorrhizien.

Ce dernier résultat, obtenu sur l'ensemble des plants, semble en contradiction avec la liaison mise en évidence sur l'échantillon de 300 plants entre la fertilisation et l'importance du polycyclisme en modalité désherbée. Mais nous avons vu (fig 5) qu'il existe une bonne relation entre le pourcentage de plants polycycliques et l'importance moyenne du polycyclisme par plant à l'échelle du placeau. Si l'on utilise cette relation pour estimer l'importance moyenne du polycyclisme par plant pour l'ensemble des placeaux, on constate que les placeaux de l'échantillon (d'écartement $2 \times 2 \mathrm{~cm}$ ) présenteraient alors une répartition de l'importance du polycyclisme entre modalités fertilisé et non-fertilisé très particulière par rapport à l'ensemble des placeaux. La relation utilisée étant peu étroite, on ne peut toutefois pas exclure totalement l'influence de la fertilisation sur l'importance du polycyclisme une fois son expression déclenchée par le désherbage.

La plus forte probabilité d'être polycyclique chez les plants de plus petit diamètre fait penser à un phénomène de compensation, puisque le polycyclisme permet un développement aérien plus important. $\mathrm{Ce}$ résultat s'oppose toutefois aux corrélations positives avec la vigueur observées par certains auteurs (Falcone et al, 1986), mais cette "vigueur» n'est pas toujours estimée initialement et peut être une conséquence du polycyclisme. De même, Reich et al (1980) font I'hypothèse que le rythme est favorisé lorsque la quantité de réserves hydro-carbonées est suffisante et que le besoin d'entretien ou de renouvellement de la structure (notamment au niveau de l'équilibre racine/tige) n'est pas très important. Dans le cadre de cette dernière hypothèse, il reste toutefois possible que les plants de plus faible diamètre, qui ont a priorimoins de racines, ont eu leur appareil racinaire proportionnellement moins «taillé» lors de l'habillage précédant la plantation, entraînant ainsi chez ces plants un rapport racine/tige plus favorable au polycyclisme. La relation polycyclisme-diamètre, qui n'existe pas pour la hauteur initiale du plant, milite, quoi qu'il en soit, en faveur d'une explication racinaire, la biomasse racinaire étant généralement assez bien corrélée au diamètre basal des plants. En effet, l'enracinement peut être la cause du non-démarrage du polycyclisme dans la mesure où seuls les plants installés, qui ont les moyens de prélever eau et éléments minéraux dans le sol, pourront exprimer leur potentiel polycyclique. Ayant disposé de matériel relativement variable sur le plan génétique (une provenance), il n'est toutefois pas possible d'écarter un déterminisme d'ordre génétique en relation avec le diamètre, compte tenu de 
I'hétérogénéité de croissance intraprovenance (Farmer, 1975).

Enfin, la relation relativement étroite obtenue au niveau placeau entre le pourcentage de plants polycycliques et le volume moyen de pousses estivales formé par plant appelle plusieurs remarques. D'une part, l'augmentation du volume moyen des pousses est principalement due à l'occurrence de plants à gros volumes, relativement rares, dans les placeaux à fort taux de polycyclisme. On peut donc penser que ces plants ont la même faible probabilité de s'exprimer dans tout placeau, et donc qu'ils ne s'expriment réellement que dans les placeaux où le nombre de possibilités (nombre de plants polycycliques) est suffisant, selon un processus purement aléatoire. Cette hypothèse est renforcée par l'existence du même type de phénomène lorsque les plants sont regroupés par classe de diamètre (tableau III), le nombre de possibilités étant constitué par l'effectif de plants par catégorie de diamètre, et l'effectif de plants à grand volume lui étant alors proportionnel. Une autre hypothèse pourrait être que les placeaux les plus riches en plants polycycliques offrent de meilleures conditions de croissance durant l'été. Mais cela n'expliquerait pas la relative rareté, même dans ces placeaux, des plants à grand volume d'été. Enfin, cette variation parallèle des 2 variables du polycyclisme peut faire penser à une origine génétique (gènes liés) ; toutefois, cette dernière hypothèse nécessite des mélanges différents et très graduels de génotypes entre placeaux, ce qui est peu plausible ici. Quoi qu'il en soit des relations, directes ou indirectes, entre ces 2 variables, elles suggèrent l'existence de 2 palliers successifs à franchir chez un plant pour exprimer son potentiel polycyclique.

\section{Conséquences du polycyclisme}

Mis à part le plus grand allongement des axes concernés par le polycyclisme, ou les conséquences sur la forme du houppier (Falcone et al, 1986), on trouve peu de références sur les autres nombreuses conséquences du phénomène pour l'appareil aérien des arbres ou des plants.

Parmentier et Barnola (1993) font l'hypothèse que l'activité cambiale est susceptible d'entrer en compétition avec l'allongement de la tige, et qu'elle s'effectue de ce fait plutôt durant les périodes de repos séparant les cycles. Les résultats de Meredieu (1993) montrent qu'en conditions de terrain la croissance en diamètre des jeunes chênes rouges a lieu en effet principalement après le premier cycle de croissance, pendant la deuxième partie de la saison de végétation (mai-septembre). Toutefois, nos résultats font apparaître (fig 6) que ce sont les plants polycycliques qui ont le plus fort accroissement en diamètre en modalité désherbée ; cet accroissement, déjà doublé chez les plants monocycliques sous l'effet du désherbage, double encore une fois chez les plants polycycliques désherbés. II serait donc intéressant de savoir si le nombre de cycles est associé seulement à une plus grande intensité de croissance en diamètre répartie de manière uniforme sur une durée relativement courte, et/ou si le plus grand nombre de cycles induit une prolongation rythmée de la croissance en diamètre en alternance avec l'élongation. Quoi qu'il en soit, l'augmentation du nombre de cycles entraîne nécessairement une augmentation de la quantité d'assimilats produits (surface foliaire augmentée) qui pourraient donc être directement utilisés pour la croissance cambiale.

L'absence de conséquences significatives du nombre de cycles de croissance sur l'accroissement en hauteur s'oppose aux résultat d'Immel et al (1978) sur Quercus prinus. Elle n'est toutefois pas surprenante si l'on remarque, avec Collin et al (1993), la tendance de l'espèce chêne rouge à former des relais subterminaux ou basaux. Ce phénomène de relais, relativement fréquent dans l'essai, a sans doute été accen- 
tué dans notre cas par les problèmes de transplantation. On a montré par ailleurs ici que le polycyclisme induit bien une augmentation de la longueur de pousses annuelles formées sur les ramifications en 1992.

Les accroissements du nombre de feuilles et de la surface foliaire par plant en relation avec le nombre de cycles ont été également notés respectivement par Igboanugo (1990) d'une part et Immel et al (1978) et Phares (1971) d'autre part. L'originalité de nos résultats est de mettre en évidence que cette importante augmentation de la surface foliaire par plant $(+118 \%)$ est due à la fois à la formation de 10 feuilles supplémentaires d'été par plant en moyenne, et à la dimension plus importante de ces nouvelles feuilles $(+64 \%)$. De ce point de vue, le polycyclisme n'apparaît pas comme un phénomène compensatoire, puisque les caractéristiques foliaires initiales des plants concernés ne diffèrent pas de celles des plants à un seul cycle.

Il constitue par conséquent un avantage considérable sur le plan photosynthétique, dont on peut se demander s'il est utilisé en totalité ou en partie pour la croissance contemporaine ou pour celle de l'année suivante (par l'intermédiaire de réserves).

Les conséquences du nombre de cycles sur l'importance de la ramification n'ont pas à notre connaissance été quantifiées globalement par d'autres auteurs, de manière quasi certaine pour le chêne rouge d'Amérique (Quercus rubra L). Seules des conséquences partielles comme l'augmentation du nombre de fourches (Falcone et al, 1986, sur le chêne rouge) ont été étudiées. Bien qu'il soit évident que le nombre de pousses nouvelles soit forcément plus important sur les branches (nombreuses) que sur l'axe principal (unique), nous avons montré que le développement estival se répartit davantage sur les ramifications par rapport au développement de printemps. Ce résultat est à relativiser dans la mesure où les plants étaient initialement pas ou peu ramifiés, et donc où le nombre de bourgeons potentiels était plus grand au début de l'été qu'au début du printemps. II n'est d'ailleurs pas possible dans le cadre de cet article de traduire le phénomène en termes de nombre de ramifications. En revanche, il a été possible de montrer que les ramifications estivales sont en moyenne plus grosses que les ramifications printanières, malgré l'«avance" de ces dernières dont l'accroissement en diamètre peut se dérouler durant la totalité de la saison. Globalement, le polycyclisme (2 ou 3 cycles) a pour conséquence une augmentation considérable du volume du houppier en modalité désherbée : $+2,3 \mathrm{~cm}^{3}$ alors que le volume de houppier des plants à un seul cycle est de $0,2 \mathrm{~cm}^{3}$.

\section{CONCLUSIONS}

Cette première étude du développement polycyclique juvénile du chêne rouge d'Amérique en conditions naturelles a permis de mettre en évidence certains «facteurs» du milieu auxquels la croissance rythmique de l'espèce est sensible. II s'agit seulement ici de facteurs composés, l'analyse n'ayant pas permis dans un premier temps d'identifier de manière certaine des composantes précises du milieu. L'action positive du désherbage prédomine largement lors de cette première année de croissance (1992). Ce résultat est à confirmer dans d'autres conditions de croissance (site et année climatique), mais il suggère dès maintenant de tester séparément l'influence de l'humidité du sol (effet négatif des excès d'eau de fin de printemps) et celle des facteurs énergétiques positifs (température et lumière), dans des conditions mieux contrôlées. La composante génétique gagnerait également à être définie de manière plus étroite (clones). Dès 1994, une étude entreprise en pépinière et en serre permettra d'une part de 
déterminer les périodes précises de l'année où sont initiés les composants des unités de croissance et d'autre part de tester l'influence d'un excès d'humidité sur l'apparition des cycles d'été.

Au niveau appliqué, I'hypothèse de l'influence du pourcentage de système racinaire initial des plants (et donc du degré d'«habillage" des plants et de leur mode d'élevage et d'arrachage en pépinière) mérite d'être étudiée attentivement. En effet, les conséquences du polycyclisme sur la production aérienne sont par ailleurs considérables. Et on peut penser que la possibilité pour un plant de développer plusieurs cycles dans l'année représente un avantage très important qui pourrait assurer son avenir proche, et en tout cas limiter le risque de mortalité dès la première année. Cette remarque vaut également pour le degré d'entretien de la plantation dès les premières années.

Nous donnerons dans un prochain article les résultats obtenus sur le même essai lors de la deuxième année de croissance (1993). Ils permettront probablement de faire un premier tri dans les hypothèses avancées pour comprendre le fonctionnement polycyclique des plants. D'autre part, nous étudierons l'influence du milieu sur la forme et l'architecture fine des plants prise au point de vue qualitatif.

\section{REMERCIEMENTS}

Cette étude a été réalisée grâce aux concours financiers de la région Midi-Pyrénées et de la CEE (contrat AIR3-CT 920134).

Nous sommes redevables à $D$ Barthélemy pour sa participation active et ses réflexions concernant la description de l'architecture des plants par unité de croissance. $L$ Pagès, ainsi que $D$ Auclair, ont fourni des remarques très constructives sur la première version du manuscrit. $F$ Lagane, A Lardit et $P$ Rossetto ont réalisé les mesures et l'entretien du dispositif en 1992.

\section{RÉFÉRENCES}

Barthélémy D, Caraglio Y (1991) Modélisation et simulation de l'architecture des arbres. Forêt-Entreprise $73,28-39$

Borchert $R$ (1975) Endogenous shoot growth rhythms and indeterminate shoot growth in oak. Physiol Plant 35, 152-157

Chambers JM, Hastie TJ (1992) Statistical models in S. Wadsworth \& Brooks / Cole Advanced Books \& Software, Pacific Grove, USA

Champagnat $P$, Payan $E$, Champagnat $M$, Barnola $P$, Lavarenne S, Bertholon C (1986) La croissance rythmique de jeunes chênes pédonculés cultivés en conditions contrôlées et uniformes. In : L'arbre, compte rendu du colloque international "l'Arbre", 9-14 septembre 1986, Montpellier, France, Naturalia monspeliensia hs, 303-337

Collin P, Badot P. Millet B (1993) Étude comparée de la croissance et du développement du frêne commun (Fraxinus exceisior $\mathrm{L}$ ) et du chêne rouge d'Amérique (Quercus rubra $L$ ) cultivés en conditions contrôlées. In : Le rythme de croissance, base de l'organisation temporelle de l'arbre, colloque «Le groupe d'étude de l'arbre, 25-26 mars 1993, Angers, 74-96

Crabbé J (1987) Aspects particuliers de la morphogenèse caulinaire des végétaux ligneux et introduction à leur étude quantitative. Institut pour l'encouragement de la recherche scientifique dans l'industrie et l'agriculture, Bruxelles, Belgique

Dagnélie P (1984) Principes d'expérimentation. Les presses agronomiques de Gembloux, Gembloux, Belgique

Duchaufour $P$ (1970) Précis de pédologie. Masson, Paris

Dupré S, Thiébaut B, Teissier du Cros E (1986) Morphologie et architecture des jeunes hêtres (Fagus sylvatica $L$ ). Influence du milieu, variabilité génétique. Ann Sci For 43, 85-102

Édelin C (1983) Aspects morphologiques de la croissance rythmique chez les arbres tropicaux. In : Le rythme de croissance, base de l'organisation temporelle de l'arbre, colloque du groupe d'étude de l'arbre, 25-26 mars 1993, Angers, 13-23

Falcone P, Keller R, Le Tacon F, Oswald H (1986) Facteurs influençant la forme des feuillus en plantation. Rev For Fr 38, 315-323

Farmer RE (1975) Growth and assimilation rate of juvenile northern red oak: effects of light and temperature. For Sci 21, 373-381

Gordon DR, Rice KJ (1993) Competitive effects of grassland annuals on soil water and blue oak (Quercus douglasii) seedlings. Ecology 74, 68-82

Guard AT, Postlethwait SN (s d) Relation of the formation of annual rings to multiple flushes of growth in several species of Quercus. Indiana Acad Sci Bot 104,106 
Heller R (1968) Manuel de statistique biologique. Gauthier-Villars, Paris

Igboanugo ABI (1990) Effects of shading on shoot morphology, wood production and structure of Quercus petraea seedlings. For Ecol Manage 38, 27-36

Immel MJ, Rumsey RL, Carpenter SB (1978) Comparative growth responses of northern red oak and chesnut oak to varying photoperiods. For Sci 24 , 554-560

Kleiner KW, Abrams MD, Schultz JC (1992)The impact of water and nutrient deficiencies on the growth, gas exchange and water relations of red oak and chestnut oak. Tree Physiol 11, 271-287

Kremer A (1981) Déterminisme génétique de la croissance en hauteur du pin maritime (Pinus pinaster Ait). I. Rôle du polycyclisme. Ann Sci For 38, 199-222

Le Roux Y, Pagès L, Leconte A (1993) Synchronismes entre processus de développement aérien et racinaire chez de jeunes semis d'hévéa. In : Rythmes biologiques: de la cellule à l'homme, actes du congrès GERB 92, 25-26 mai 1992, Paris, 57-66

Meredieu C (1993) Installation de parcelles agroforestières : croissance du chêne rouge d'Amérique (Quercus rubra $L$ ) en relation avec un enherbement spontané. Mémoire, École supérieure d'agriculture d'Angers, Angers

Millet B, Bonnet B, El-Morsy AW (1991) Le fonctionnement rythmique des végétaux ligneux. In : L'Arbre.
Biologie et développement ( $C$ Édelin, éd), Naturalia monspeliensia hs 295-317

Parmentier C, Barnola P, Maillard P, Lavarenne S (1991) Ėtude de la croissance rythmique du chêne pédonculé, influence du système racinaire. In : L'Arbre. Biologie et développement (C Édelin, éd), Naturalia monspeliensia hs, 328-343

Parmentier C, Barnola P (1993) Quelques aspects du rythme endogène de la croissance chez les arbres tempérés. Approche de son déterminisme. In : Le rythme de croissance, base de l'organisation temporelle de l'arbre. colloque du groupe d'étude de l'arbre, 25-26 mars 1993, Angers, 63-73

Phares RE (1971) Growth of red oak (Quercus rubra L) seedlings in relation to light and nutrients. Ecology 52 , 669-672

Reich PB, Teskey RO, Johnson PS, Hinckey TM (1980) Periodic root and shoot growth in oak. For Sci 26 , 590-598

Sprent $P$ (1993) Pratique des statistiques non-paramétriques (traduction française JP Ley). INRA, collection Techniques et Pratiques, Paris

Smethurst PJ, Comerford NB, Neary DG (1993) Predicting the effects of weeds on $K$ and $P$ uptake by young slash pine on a spodosol. Ecol Manage 60 . 27-39

White J (1979) The plant as a metapopulation. Ann Rev Ecol Syst 10, 109-145 